\title{
ERS International Congress, Madrid, 2019: highlights from the Basic and Translational Science Assembly
}

Niki D. Ubags ${ }^{1}$, Jonathan Baker ${ }^{2}$, Agnes Boots $^{3}$, Rita Costa ${ }^{4}$, Natalia El-Merhie $\mathbb{C}^{5}$, Aurélie Fabre ${ }^{6,7}$, Alen Faiz ${ }^{8}$, Irene H. Heijink ${ }^{9}$, Pieter S. Hiemstra (10 ${ }^{10}$, Mareike Lehmann ${ }^{4}$, Silke Meiners ${ }^{11}$, Sara Rolandsson Enes ${ }^{12,13}$ and Sabine Bartel (109

\begin{abstract}
Affiliations: ${ }^{1}$ Faculty of Biology and Medicine, University of Lausanne, Service de Pneumologie, CHUV, Lausanne, Switzerland. ${ }^{2}$ Airway Disease Section, National Heart and Lung Institute, Imperial College London, London, UK. ${ }^{3}$ Dept of Pharmacology and Toxicology, Maastricht University, Maastricht, the Netherlands. ${ }^{4}$ Lung Repair and Regeneration Unit, Helmholtz-Zentrum Munich, Ludwig-Maximilians-University, University Hospital Grosshadern, Member of the German Center of Lung Research (DZL), Munich, Germany. ${ }^{5}$ Early Life Origins of Chronic Lung Disease, Research Center Borstel, Leibniz Lung Center, Member of the DZL and the Airway Research Center North (ARCN), Borstel, Germany. ${ }^{6}$ St Vincent's University Hospital, Dublin, Ireland. 7 University College Dublin School of Medicine, Dublin, Ireland. ${ }^{8}$ University of Technology Sydney, Respiratory Bioinformatics and Molecular Biology (RBMB), School of Life Sciences, Sydney, Australia. 'University of Groningen, University Medical Center Groningen, Depts of Pathology \& Medical Biology and Pulmonology, Groningen, The Netherlands. ${ }^{10}$ Dept of Pulmonology, Leiden University Medical Center (LUMC), Leiden, The Netherlands. ${ }^{11}$ Comprehensive Pneumology Center (CPC), University Hospital, Ludwig-Maximilians University, Helmholtz Zentrum München, Member of the DZL, Munich, Germany. ${ }^{12}$ University of Vermont, Dept of Medicine, Larner College of Medicine, Burlington, VT, USA. ${ }^{13}$ Lund University, Dept of Experimental Medical Science, Lund, Sweden.
\end{abstract}

Correspondence: Sabine Bartel, University Medical Center Groningen, Hanzeplein 1, 9700 RB Groningen, The Netherlands. E-mail: s.r.barteldumcg.nl

ABSTRACT In this review, the Basic and Translational Sciences Assembly of the European Respiratory Society (ERS) provides an overview of the 2019 ERS International Congress highlights. In particular, we discuss how the novel and very promising technology of single cell sequencing has led to the development of a comprehensive map of the human lung, the lung cell atlas, including the discovery of novel cell types and new insights into cellular trajectories in lung health and disease. Further, we summarise recent insights in the field of respiratory infections, which can aid in a better understanding of the molecular mechanisms underlying these infections in order to develop novel vaccines and improved treatment options. Novel concepts delineating the early origins of lung disease are focused on the effects of pre- and post-natal exposures on neonatal lung development and long-term lung health. Moreover, we discuss how these early life exposures can affect the lung microbiome and respiratory infections. In addition, the importance of metabolomics and mitochondrial function analysis to subphenotype chronic lung disease patients according to their metabolic program is described. Finally, basic and translational respiratory science is rapidly moving forward and this will be beneficial for an advanced molecular understanding of the mechanisms underlying a variety of lung diseases. In the long-term this will aid in the development of novel therapeutic targeting strategies in the field of respiratory medicine.

@ERSpublications

Highlights of basic and translational science presented at \#ERSCongress 2019 summarising latest research on the lung cell atlas, lung infections, early origins of lung disease and the importance of metabolic alterations in the lung http://bit.ly/2UbdBs4

Cite this article as: Ubags ND, Baker J, Boots A, et al. ERS International Congress, Madrid, 2019: highlights from the Basic and Translational Science Assembly. ERJ Open Res 2020; 6: 00350-2019 [https://doi.org/10.1183/23120541.00350-2019]. 


\section{Introduction}

The European Respiratory Society (ERS) International Congress 2019 in Madrid brought together leading experts in all fields of respiratory medicine and research. The Basic and Translational Sciences Assembly organised three scientific symposia and two hot topics sessions and received 351 abstracts for the congress in Madrid, from which 336 were accepted and presented by early career members. In line with [1], we here summarise a selection of major scientific highlights that were discussed in Madrid 2019.

\section{The human lung cell atlas}

Single cell RNA sequencing (scRNA Seq) is a rapidly developing technology, enabling researchers to delineate gene expression patterns of individual cells. This technology is key for the so-called human lung cell atlas, a dynamic online platform freely accessible to the scientific community (lungcellatlas.org). The first presentation in this session was given by Martijn Nawijn (University Medical Center Groningen, Groningen, The Netherlands) about using sc-Seq technology to investigate the differences between childhood onset asthmatics and healthy controls in human bronchial biopsies [2]. Single cells dissociated from airway wall biopsies were analysed using a Droplet-based 10x Genomics (Pleasanton, CA, USA) platform. Early results were biased towards an over-representation of airway epithelial cells compared to the numbers seen in histology of adjacent biopsies. Comparison between disease status showed an expected increase in the number of goblet cells in the asthmatic airways as well as a new type of differentiated cell named "mucous ciliary cell", that expresses both mucin and ciliary genes. To determine the origin of this new cell type, linkage trajectory analysis was conducted, which found basal to club to ciliated or to mucus cell trajectories. This mucous ciliated cell state was identified in asthmatics, with active transitions between ciliated and goblet cells. T-helper cells type 2 (Th2-cells) were also found to be increased in the asthmatic biopsies. Using cell-cell interaction analysis, using the receptor expression by one cell and expression of the cytokine/chemokine from another; there was a shift from airway structural cell communication in healthy lungs to a Th2-dominated interactome in asthma. This indicates that increased mucus expression in asthma is driven by the interactions with Th2 cells through interleukin (IL)-13 signalling and this Th2-dominated interactome might be a good candidate for potential novel therapeutic targets.

Next, Pascal Barbry (Institut de Pharmacologie Moléculaire et Cellulaire, Valbonne, France) presented two complementary airway in-vitro and in-vivo models: 1) a 3D model of primary airway culture of airway epithelial cells grown at air liquid interface (ALI) to study mechanics of differentiation; and 2) data obtained from bronchoscopy samples across different regions of the lung. ALI- grown epithelial cell differentiation was assessed at days 7, 12, 28 and 47 of air exposure, then ran on the 10x platform. Using t-distributed stochastic neighbour embedding ( $t$-SNE) plots based subpopulations of cells, scRNA expression patterns were resolved over time. A large population of airway epithelial cells with proliferative signature was observed at the early time point of differentiation, but diminished at later time points. Using the sample approach different cell types (such as basal and goblet cells) can be followed over the differentiation process. Using this method, an overlap between the secretory/club and goblet cells was identified, with an overlap between the two cell types for a number of specific markers. Furthermore, a new type of airway cell was described, the "deuterosomal cell", a precursor between secretory and multiciliated cells, similar to Martijn Nawijn's work. However, here, this cell type transition was also found in healthy tissue. Using linkage trajectory analysis it was predicted that club cells act as precursors of multiciliated cells and goblet cells. In addition, goblet cells may also act as precursors of multiciliated cells.

A new tissue dissociation method using a cold-active protease from Bacillus licheniformis was also described, which allows for dissociation at cold temperatures limiting the degradation of RNA and resulting in the collection of $80-90 \%$ viable cells [3]. By comparing gene expression throughout the airway (nose, trachea, bronchus (5-6 division) and distal bronchus), a close relationship was found between the trachea and bronchus, and certain cell subpopulations were specific to one region, such as macrophages to the distal lung samples.

Joachim Schultze (University of Bonn, Bonn, Germany) presented his unpublished research on chronic obstructive pulmonary disease (COPD) and scRNA-seq. This study used multi-colour flow cytometry for identification and characterisation of immune cells derived from blood and bronchoalveolar lavage (BAL) of control and Global Initiative for Chronic Obstructive Lung Disease (GOLD) stage II COPD patients. Due to the challenges in single cell analysis regarding cell viability and survival in droplet based methods, a comparative analysis between a droplet-based (10x Genomics), with a gravity-based method (Seq-Well), of scRNA-seq was performed. Mathematical integration of data obtained from samples of different controls and patients allowed unbiased clustering of cells based on common expression profiles and additional background cell-specific information. Machine learning-assisted cell annotation algorithms are able to calculate the cell label probability closest to known literature. The success of this approach was 
demonstrated by confirming that neutrophils [4] and type 1 innate lymphoid cells are increased in COPD samples [5]. After establishing, optimisation and validation of the bioinformatics analysis, myeloid cell populations, more specifically neutrophils, monocytes and macrophages, were found to be under-represented in the droplet-based scRNA-seq. The gravity-based method showed a higher representation and annotation of immune cells. For example, three different clusters of neutrophils as well as multiple alveolar macrophage populations were identified; this could both account for different cell types or different cell states. Regarding differences between COPD and control samples, proliferating macrophages as well as monocyte derived macrophages were increased in COPD, while macrophages expressing genes involved in antigen processing and presentation as well as cell motility were decreased. Confirmatory downstream studies showed a reduction in MHC class I and II molecules as well as reduced macrophage motility in cells isolated from COPD patients. Heterogeneous but significant changes in lipid metabolism were also present in the macrophages from COPD patients, in particular cholesterol metabolism. In conclusion, differences in cell plasticity allow the identification of disease- and patient-specific changes, which could explain heterogeneity in the pathophysiology of COPD.

Finally, Herbert Schiller (Comprehensive Pneumology Center Munich) presented longitudinal scRNA-seq data for cell fate trajectory studies focusing on defective repair mechanisms and regeneration responses after lung injury. scRNA-seq data from bleomycin injured mouse lung tissue were used to identify the evolution of different cell types and states which could predict cell dynamics and explain human variations observed when comparing control and end-stage lung fibrosis tissue samples. Differences in receptor-ligand pairs predicted active cell communications over time, calculating relative proportions of altered interactions, such as macrophages-endothelial cells as well as fibroblasts-alveolar epithelium communications. During homeostasis, mesenchymal cell populations showed heterogeneity in the alveolar type II cell niche. It was possible to characterise matrixfibroblasts, lipofibroblasts, pericytes, Hedgehog-interacting protein-positive (Hhip+) fibroblasts and smooth muscle cells and to confirm this profile by spatial validation using multiplexed single molecule in situ mRNA hybridisation [6, 7] together with surfactant protein $\mathrm{C}$ fluorescent staining. During fibrogenesis, lipofibroblasts expressing transcription factor 21 (Tcf21) appeared to be the major source of injury-induced myofibroblast in the mouse. Mouse and human data integration showed human fibroblast subtype correspondence, but Hhip+ fibroblasts were not matched to any of the human mesenchymal populations. Regarding injury specific alveolar epithelial cell states, high-resolution real-time scRNA-seq revealed a squamous transient keratin 8 positive (Krt8+) alveolar differentiation intermediate cell type [8] present only during the bleomycin fibrogenesis phase. The state of these cells was further assessed by RNA velocity [9]. Sequential sampling over time allows the differentiation between unspliced and spliced mRNA, distinguishing between newly synthesised versus mature mRNA. Using this tool, Krt8+ cells were predicted to be derived from alveolar type II cells and a new airway club cell population was characterised by very high expression of MHC class II molecules. Traditional lineage tracing experiments further confirmed the dual origin prediction from single cell analysis. Pseudotime trajectories showed that intermediate Krt8+ cells differentiate into terminal alveolar type I cells. Further use of human precision-cut lung slices and organoids will allow the induction of perturbations in the different cell populations to validate and help with new findings relevant to degenerative lung diseases.

Finally, scRNA-seq is a promising, rapidly developing novel technology, enabling researchers to gain important insight into cellular trajectories in lung health and disease. Furthermore, as evident from these studies, this technology can identify novel/rare cell types in the lung, which then need to be validated and further understood using other methods such as immunofluorescence staining.

\section{Scientific year in review: respiratory infections}

This year's "Scientific Year in Review" session opened with the ceremony for the Romain Pauwels Research Award. In 2019, this award presented by Glaxo Smith Kline (€ 50000) was given to Klaus Bønnelykke (Copenhagen University, Copenhagen, Denmark) for his achievements in the field of paediatric asthma. His research is focused on the early origins and genetic determinants of asthma, allergy and eczema, and has resulted in identification of susceptibility genes and other risk factors for these diseases. He performed the first genome-wide association study of severe asthma exacerbations in early childhood, showing the relevance of phenotyping for identification of mechanisms [10]. This study identified CDHR3 as a susceptibility gene, and later Dr Bønnelykke provided evidence that the gene product of $C D H R 3$ functions as a rhinovirus $C$ receptor [11]. Furthermore, he is co-principal investigator of the COPSAC2010 birth cohort.

Following the award ceremony, four expert speakers discussed the newest research on respiratory infections. Simon Jochems (Leiden University Medical Center, Leiden, The Netherlands) covered the innate and adaptive immune responses against Streptococcus pneumoniae, the most common cause of 
pneumonia in humans. Over 100 serotypes are currently known, and $50 \%$ of children and $10 \%$ of adults are colonised with $S$. pneumoniae [12], protecting them from infection due to pre-existing immunity. Dr Jochems uses the pneumococcal challenge model, a controlled infection of healthy volunteers with 80000 colony forming units (cfu) of S. pneumoniae via the nostrils. As bacterial pneumonia is one of the main causes of death after influenza virus infection, the group exposed healthy volunteers to either saline fluid or live attenuated influenza vaccine (LAIV) 3 days prior to the S. pneumoniae application and investigated the resulting anti-bacterial immune response. The LAIV pre-application induced a transient, 10 -fold increase in the $S$. pneumoniae density in the nose. Nasal cytokine measurements revealed that LAIV treatment alone caused high inflammation, while $S$. pneumoniae application did not [13]. Increased levels of Interferon gamma-induced protein after LAIV were found to be associated with a higher $S$. pneumoniae density and were predictive of $S$. pneumoniae colonisation, while the opposite was observed for IL-10. Furthermore, LAIV application impaired neutrophils' ability to kill invading S. pneumoniae, and monocyte invasion in the nose to clear the infection, resulting in a higher bacterial density of S. pneumoniae. This could explain why bacterial infections after influenza are more severe.

In a second study the group investigated nasal biopsies after $S$. pneumoniae challenge of healthy volunteers to find immune markers that stratify protected patients versus non-protected ones [14]. The biopsies were analysed by CyTOF mass cytometry, allowing up to 38 cellular markers to be analysed on single cell suspensions. Seven distinct immune cell clusters (three B-cell clusters and four CD8+ T-cell clusters) were higher in patients who were protected from S. pneumoniae colonisation and thus may serve as predictive markers in the future.

Simone Joosten (Leiden University Medical Center) discussed innate immune memory to improve respiratory health. The innate immune system has classically been thought to be non-specific, and lacking the potential to build up immunological memory. However, this dogma has recently been challenged by several studies using the Bacillus Calmette Guérin (BCG) vaccine [15-17]. BCG vaccination modulates non-specific immune responses against many pathogens, not only tuberculosis [18]. The exact mechanisms of this heterogenic and incomplete protection are still unknown $[19,20]$. In mice BCG vaccination has the potential to protect against secondary infections in addition to mycobacteria [21-23], which has also been observed in newborn children [24, 25]. Growing literature suggests that the resistance to secondary infections after BCG vaccination cannot only be attributed to the adaptive immune system. Dr Joosten presented a study in which the BCG vaccine was shown to induce expansion of haematopoietic stem cells (HSCs) as well as to induce changes in their gene expression in the bone marrow. These modified HSCs generated "trained" monocytes/macrophages that possessed enhanced anti-mycobacterial capacity [15]. Further, respiratory viral infection can induce long-lasting memory in alveolar macrophages [26]. The exact mechanisms are currently unknown, however, Simone Joosten highlighted several different pathways $[27,28]$. She explained that a "defence ready state" includes high glycolysis, increased expression of cytokines, MHC II, defence genes and increased effector responses (e.g. bacterial killing). Although big steps towards understanding the exact mechanism of BCG-induced trained immunity have been made, there is still a big knowledge gap and further studies are needed. Eventually, training of innate immunity may be employed in therapeutic strategies [29].

Sebastian Johnston (Imperial College London, London, UK) completed the session by presenting research on the interaction of bacterial and viral infections during COPD exacerbations. As severe exacerbations are responsible for decreased survival rates among COPD patients, it is critical to develop new and better prevention strategies [30]. Historically, the importance of viral and bacterial infections in precipitating COPD exacerbations has been underestimated [31]. More recently, it was found that bacterial infections, and possibly more importantly, viral infections (detected in 52\% of patients with acute exacerbations) are associated with exacerbations and that viral infections can potentially trigger secondary bacterial infections [32-34]. Sebastian Johnston suggested that increased airway glucose levels detected in COPD patients, which were further increased during acute exacerbations, may be a mechanism through which rhinoviruses enhance bacterial growth [33]. COPD patients treated with inhaled corticosteroids (ICS) have an increased risk of pneumonia and Sebastian Johnston presented that ICS treatment resulted in suppressed immune responses, including decreased levels of interferons and secretory leukocyte protease inhibitor (SLPI), impaired virus control, increased bacterial load, and increased mucin levels upon rhinovirus infection [35]. Treatment of mice with exogenous interferon- $\beta$ reversed the bacterial load and restored anti-viral activity [35]. ICS treatment also altered the lower respiratory microbiota, impairing pulmonary bacterial control in both human and mouse models, in which cathelicidin/LL-37 was shown to play a central role [36]. LL-37 levels were lower in sputum from COPD patients and negatively correlated with exacerbation severity and bacterial load. Taking together, viral and bacterial infections in COPD patients may play a greater role in triggering acute exacerbations than previously thought, and specific treatments such as interferon- $\beta$, anti-viral treatment or cathepsin D inhibitors may potentially help to prevent acute COPD exacerbations. 
In the concluding panel discussion, the importance of using experimental infection models, and analysing samples from different locations within the upper and lower respiratory tract, including nasal samples and concentrated BAL fluid samples, and the timing of sampling were discussed. Other challenges such as investigating the interaction between adaptive and innate immunity, utilising available omic-platforms, were mentioned. In summary, this session showed that there is an urgent need to better understand the pathogenesis and underlying molecular mechanisms of infections of the airways and of viral/bacterial interactions, to develop effective vaccines and novel treatments. As evidenced above, this knowledge can help to understand and maybe even prevent infectious exacerbations of lung diseases.

\section{Early origins of lung disease: an interdisciplinary approach}

This session focused on the mechanisms underlying healthy and abnormal early lung development as well as the impact of immune maturation and the microbiome on lung health and disease.

Alan Jobe (Cincinatti Children's Hospital, Cincinatti, OH, USA) presented an overview on the impact of pre- and postnatal exposures on fetal and neonatal lung development. Pre-conceptional, fetal and early childhood factors all influence lung development. These factors include epigenetic modifications, nutrition, toxic exposures, lung and gut microbiome and other specific exposures related to pregnancies [37]. Growth-restricted infants have significantly lower lung function at 8-9 years of age compared with term infants of normal birthweight [38]. It was also shown that infants who were small relative to their gestational age (defined as fetal weight or birth weight $<10$ th percentile) had higher mortality rates [39] and increased bronchopulmonary dysplasia (BPD) [40]. Prematurity is associated with increased risk for asthma, pneumonia and acute bronchiolitis [41, 42]. A recent meta-analysis showed that very preterm infants $(<1500 \mathrm{~g})$ have lower IQ values than term infants [43] which could be related to dysregulated maturation following preterm delivery.

Over the past 40 years, off-target exposures of fetus to steroids have occurred during pre-viable deliveries ( $<24$ weeks), 24-34 weeks of gestation, 34-36 weeks of gestation (according to the recommendations in the USA but not in Europe) and elective C-section delivery. These off-target exposures may negatively affect lung function. While inducing early lung maturation, antenatal steroids delay alveolarisation, leading to shrinkage of the mesenchyme before the surfactant level is increased. In order to understand which steroid exposure will stimulate lung maturation, Alan Jobe's group has used sheep and in monkey models [44, 45]. It was concluded that different outcomes, e.g. inflammatory responses, depend on the time of exposure to corticosteroids, and that these outcomes could be ablated by early induction of inflammation, in a similar process as induction of tolerance to inflammatory stimuli.

Anne Hilgendorff (Comprehensive Pneumology Center Munich) covered two different aspects of premature lung ageing: organ and cellular function. With respect to organ function, physiologic lung ageing is characterised by chest wall and thoracic spine deformities, senile emphysema, reduced respiratory muscle strength, increased alveolar dead space, functional changes of the airway receptors (response to drugs decreases) and diminished ventilator response to hypoxia and hypercapnia [46]. Therefore, physiologic lung ageing has to be taken into account when BPD infants are ageing. BPD relates to additional features of COPD as was shown by magnetic resonance imaging, where lung structural changes similar to COPD and lung fibrosis were observed in preterm infants with BPD [47]. Moreover, there is severe alteration of vasculature, preceding even the mildest forms of COPD [48], suggesting BPD will develop into COPD in later life. The underlying mechanisms leading to accelerated ageing in the COPD lung are however still poorly understood.

Remarkably there are various hallmarks of cellular ageing seen in BPD, such as inflammation, stem cell exhaustion, telomere dysfunction, epigenetic changes and senescence [46, 49, 50]. Anne Hilgendorff s group showed that prenatal smoke exposure induced a vast influx of monocytes into the lungs of fetal mice and this led to severe matrix changes. Another study demonstrated that neonatal mice receiving mechanical ventilation with oxygen-rich gas $\left(\mathrm{MV}-\mathrm{O}_{2}\right)$ had increased rates of endothelial cell apoptosis, which was aggravated in mice heterozygous (lacking one allele) for the growth factor platelet-derived growth factor receptor alpha (PDGF-Ra). Vascular and alveolar defects were due to reduced PDGF-Ra expression, activation of which supports endothelial survival [51]. Another factor involved in BPD pathophysiology is the monocytic receptor SIGLEC14, which has also been associated with increased risk of COPD exacerbation [52]. Besides inflammation, stem cell exhaustion may affect postnatal lung maturation and development, where mesenchymal stem cells fail to form the niche properly in BPD [53]. Exhaustion of airway basal progenitor cells has been implicated in early and established COPD [54]. Further, children with BPD have shorter telomere length, a biological ageing indicator, which has been linked to reduced lung function [55]. Despite these similarities, it is still unclear how premature lung ageing relates to lung disease. We need to understand which cells are over-proliferative and which ones are apoptotic, and which mechanisms to stop and which ones to enable. 
Clare Lloyd (Imperial College London) reviewed the influence of early life pulmonary immunity on the development of paediatric inflammatory respiratory disease. Severe therapy-resistant asthma (STRA) in children is characterised by poor disease control despite maximal treatment doses and optimal assessment of modifiable factors. Although allergic asthma is generally considered a Th2-mediated disease, emerging evidence suggests that non-Th2 mechanisms may contribute, specifically to severe disease. Paediatric STRA is characterised by high pulmonary interleukin (IL)-33 levels despite maximal treatment with steroids [56]. Prof. Lloyd's group recently described the role of innate lymphoid cells (ILCs) in paediatric patients with severe asthma. Type 2 ILCs (ILC-2) expressing the prostaglandin receptor CRTH2 and IL-13 were found to be increased in the sputum of paediatric STRA patients compared to non-asthmatic controls and paediatric patients with difficult asthma (i.e. poor control despite maximal treatment doses) [57]. Although CRTH2+ IL-13+ ILCs were increased in STRA at baseline, they decreased following high-dose systemic steroid treatment, suggesting that these cells are steroid responsive. ILC2s mainly accumulate in the peribronchial and perivascular areas of the lung following an acute inflammatory exposure [58]. Moreover, ILC2s were proven to move faster and over longer distances compared to CD4+ T-cells. The ILC2 motility in the lung was promoted by extracellular matrix proteins, such as collagen-I, collagen-IV and fibronectin. Collagen-I was shown to influence the shape of the ILC2 towards a pro-migratory exploratory phenotype that is critical for the regulation of eosinophilic inflammation.

Debby Bogaert (University of Edinburgh, Edinburgh, UK) discussed the important concept that age matters in the development of early life pulmonary immunity. The age at which an individual is exposed to a certain stimulus may determine how the immune response develops. The respiratory microbiome, which starts to develop from birth onwards [59], may play a crucial role and is influenced by a variety of host and environmental factors in early life [60]. Debby Bogaert presented data from a prospective birth cohort study (Microbiome Utrecht Infant Study (MUIS)) demonstrating that decreased stability of nasopharyngeal microbial communities in the first 3.5 months of life is associated with an increased susceptibility to respiratory tract infections (RTI) in the first year of life [61]. The presence of a virus (even asymptomatic) before the first month of life was associated with earlier and recurrent infections. Analysis of paired nasopharyngeal and oropharyngeal samples from the MUIS study cohort from birth until 6 months of age revealed a significant overlap in bacterial composition between the two different locations directly after birth, suggestive of similar source communities [62]. Following the first week of life, the developmental trajectories of the oropharynx and nasopharynx niches rapidly differed and became populated by niche-specific communities over time. The nasopharyngeal microbiome remains the key community that drives respiratory health and infections [61], since oral microbiome composition at 1 month of life was not predictive for the number of RTI in the first year of life [62]. Analysis of the topographical differentiation between oropharynx and nasopharynx revealed a gradual increase over the first 3 months of life. A complete loss of topographical differentiation was observed during RTI, suggesting the niches to become more similar. In order to predict severity of lower RTI (LRTI) in children, the group of Debby Bogaert developed a prediction model using machine learning on samples (paired nasopharyngeal and endotracheal aspirates) and data obtained from the paediatric intensive care unit cohort [63]. A combination of both viral and bacterial biomarkers with host factors in a sparse random forest analysis allowed for a high classification accuracy of LRTI samples, which were compared to samples from healthy controls. Important disease predictors were presence of respiratory syncytial virus, high abundance of Haemophilus influenza, H. haemolyticus, S. pneumoniae and Pseudomonas fluorescens and low abundance of Moraxella species, antibiotic treatment in the preceding 6 months and lack of breastfeeding [63]. These results suggest that the potential use of a microbiota-based diagnostic or classification tool, after validation in independent cohorts, could be useful for clinical applications in the future.

Taken together, early life (pre- and postnatal) exposures and their effects on lung development may set the stage for (chronic) respiratory disease development in later life. Furthermore, microbiota analysis and the identification of viral and bacterial biomarkers for the prediction of respiratory infections in early life can lead to the development of diagnostic or classification tools, possibly contributing to prevention of respiratory infections in early life.

\section{Metabolic alterations in lung ageing and disease}

Metabolomics is a powerful technology capturing metabolic changes in health and disease. The advantage lies in its ability to integrate the crosstalk of multiple compartments. Craig Wheelock (Karolinska Institute, Stockholm, Sweden) discussed the so-called "metabotype" which is influenced by genome, microbiome and exposome (representing the sum of all expositions), thereby creating a snapshot of the patient's phenotype. The metabotype has the potential to discriminate health and disease states as well as distinct clinical phenotypes. It has been analysed within the U-BIOPRED study in which the molecular phenotype of severe asthma was investigated in an adult and a paediatric cohort. A cluster of urine metabolites was 
used to distinguish severe and mild asthma from healthy controls. In severe asthmatic patients, this metabotype is stable upon longitudinal sampling. Comparing serum and urine metabolome revealed metabolically distinct subphenoytpes for moderate and severe asthmatics influenced by steroid treatment [64], indicating that the metabotype is sensitive to therapeutic regiments. Metabotypes vary in different chronic lung diseases such as COPD [65] and BPD with similar biochemical and metabolic shifts in both sexes, but much stronger in females than males. Many of the detected metabolites in metabolomics are microbiome-based, thus understanding the gut-lung axis is vital to decipher the role of the metabolites in the development of lung disease.

Peter Barnes (Imperial College London) reviewed the idea that COPD can be regarded as a disease of accelerated ageing including metabolic alterations associated with elevated levels of cellular senescence [66]. Senescent cells remain metabolically active, secreting a mixture of pro-inflammatory cytokines, chemokines and proteases, known as the senescence-associated secretory phenotype, mirroring the inflammatory secretome in COPD [67]. Removal of senescent cells of the lung using senolytics, which induce apoptosis in senescent cells, might be a promising therapeutic approach for COPD as indicated by the first human trial in IPF [68].

Ageing and COPD are also characterised by mitochondrial alterations with increased mitochondrial mass in the aged and COPD lung due to impaired mitophagy upon dysregulation of the PINK1/Parkin axis, resulting in elevated numbers of fused and swollen mitochondria [69]. Basal and maximal respiration of mitochondria are reduced in smooth muscle cells from COPD patients [70]. Aged mitochondria can induce activation of the inflammasome via release of their DNA [71].

Philip Hansbro (University of Technology Sydney, Sydney, Australia) presented data on altered immunometabolism and mitochondrial dysfunction in COPD, using a novel mouse model of intranasal cigarette smoke exposure [72]. Damaged mitochondria release mitochondrial reactive oxygen species (mROS) [73], which may further exacerbate accelerated ageing and mitochondrial damage. Usually, mROS, a major by-product of oxidative phosphorylation, are removed by antioxidants such as hydrogen sulphide $\left(\mathrm{H}_{2} \mathrm{~S}\right)$ [73]. $\mathrm{H}_{2} \mathrm{~S}$ is an endogenous mitochondrial specific antioxidant, which is both mitochondrial protective and anti-inflammatory [74]. $\mathrm{H}_{2} \mathrm{~S}$ has been reported to be reduced in both human COPD and COPD mouse models $[75,76]$, suggesting that the loss of this antioxidant contributes to disease pathology. Novel $\mathrm{H}_{2} \mathrm{~S}$ donors, such as AP39 and RT-O, reduced cigarette smoke-induced ROS levels in vitro in an epithelial ALI culture model and reduced inflammatory cell numbers in the lung and markers of fibrosis in vivo in intranasally smoked mice. Treatment with other antioxidants, such as the mitochondrial-targeted peptide SS-31 and the Nrf2-activating itaconate, showed similar protective effects $[71,72,74-77]$. Thus, mROS scavengers, including mitoQ, SKQ and mitoTempo, may be considered as new potential therapeutics for COPD treatment $[70,78]$.

An important cellular player regarding mitochondrial dysfunction and lung ageing are macrophages, which display phenotypic changes and defective phagocytosis in COPD [79]. In the intranasal cigarette smoke model, macrophages secreted higher levels of TNF- $\alpha$ and IL-1 $\beta$ and the inflammatory macrophage marker, MARCO, was elevated on the cell surface. In the early stages of the model, macrophages showed a decrease in glycolysis rates but an increase in both glycolysis and oxidative phosphorylation after 8 weeks. Inhibition of glycolysis using 2DG glucose reduced inflammation and BAL inflammatory cell infiltrates, suggesting a pathogenic role for metabolic reprogramming of macrophage in cigarette smoke-induced lung inflammation. An increase in mitochondrial mass was also observed in CD11B+ macrophages, indicating the potential presence of defective mitophagy or increased mitofusion. Understanding how dysregulated mitochondrial function contributes to proinflammatory macrophage function during COPD pathogenesis, and targeting these macrophages therapeutically, may help to reduce the inflammation present in the COPD lung.

Anna Krasnodembskaya (University of Belfast, Belfast, UK) outlined that proper functioning of macrophages and epithelial or various other cells, can be affected by mesenchymal stromal cells (MSC) transferring their mitochondria via tunnelling nanotubes or extracellular vesicles [80]. Mitochondria derived from MSCs are able to enhance the phagocytic activity of alveolar macrophages in vivo. Inhibition of the formation of tunnelling nanotubes abrogated the effects of MSCs on macrophage phagocytosis in vitro and their anti-microbial effects in vivo. However, inhibiting formation of tunnelling nanotubes in MSCs did not fully block mitochondrial transfer, suggesting an additional mitochondrial transfer mechanism via extracellular vesicles [81].

Inflammatory macrophages that had been exposed to either lipopolysaccharide or BAL fluid samples derived from acute respiratory distress syndrome (ARDS) patients, showed increased uptake of mitochondria derived from MSCs and enhanced oxidative phosphorylation and ATP production, when incubated with conditioned media from MSCs. This was dependent on the mitochondrial activity of the 
MSCs, as MSCs with defective mitochondrial respiration were no longer able to activate the respiratory function in inflammatory macrophages, which was also confirmed in an in vivo model of ARDS [82]. Taken together, within an inflammatory environment in the lung, mitochondrial transfer may lead to metabolic reprogramming of macrophages towards an anti-inflammatory phenotype with enhanced phagocytic activity.

In conclusion, metabolomics and the analysis of mitochondrial function are emerging as a powerful approach to reveal metabolic reprogramming in subtypes of chronic lung diseases. Phenotyping of subgroups of patients as well as further stratifying according to sex seems to be required to identify small changes in metabotypes and metabolic alterations, which may offer new therapeutic options for lung disease.

\section{Concluding remarks}

The basic and translational science sessions at the ERS Congress presented important scientific advances in the respiratory field. The large interest of both, basic researchers and clinical scientists highlights the importance of translational science in driving an advanced molecular understanding of lung diseases for the development of novel therapeutic targeting strategies.

Support statement: S. Bartel and S. Rolandsson Enes are supported by an ERS/EU RESPIRE 3 Marie Sklodowska-Curie Fellowship from the European Respiratory Society and the European Union's H2020 research and innovation programme under the Marie Sklodowska-Curie grant agreement number 713406.

Conflict of interest: N.D. Ubags has nothing to disclose. J. Baker has nothing to disclose. A. Boots has nothing to disclose. R. Costa has nothing to disclose. N. El-Merhie has nothing to disclose. A. Fabre has nothing to disclose. A. Faiz has nothing to disclose. I.H. Heijink has nothing to disclose. P.S. Hiemstra has nothing to disclose. M. Lehmann has nothing to disclose. S. Meiners has nothing to disclose. S. Rolandsson Enes reports an ERS/EU Marie-Curie Post-doctoral Research Fellowship (RESPIRE3) during the writing of this article. S. Bartel reports grants and personal fees from Bencard Allergie GmbH outside the submitted work.

\section{References}

1 Nikolic MZ, Garrido-Martin EM, Greiffo FR, et al. From the pathophysiology of the human lung alveolus to epigenetic editing: Congress 2018 highlights from ERS Assembly 3 "Basic and Translational Science". ERJ Open Res 2019; 5: 00194-2018.

2 Vieira Braga FA, Kar G, Berg M, et al. A cellular census of human lungs identifies novel cell states in health and in asthma. Nat Med 2019; 25: 1153-1163.

3 Ruiz Garcia S, Deprez M, Lebrigand K, et al. Novel dynamics of human mucociliary differentiation revealed by single-cell RNA sequencing of nasal epithelial cultures. Development 2019; 146: dev177428.

4 Gunay E, Sarinc Ulasli S, Akar O, et al. Neutrophil-to-lymphocyte ratio in chronic obstructive pulmonary disease: a retrospective study. Inflammation 2014; 37: 374-380.

5 Silver JS, Kearley J, Copenhaver AM, et al. Inflammatory triggers associated with exacerbations of COPD orchestrate plasticity of group 2 innate lymphoid cells in the lungs. Nat Immunol 2016; 17: 626-635.

6 Chen KH, Boettiger AN, Moffitt JR, et al. RNA imaging. Spatially resolved, highly multiplexed RNA profiling in single cells. Science 2015; 348: aaa6090.

7 Raj A, van den Bogaard P, Rifkin SA, et al. Imaging individual mRNA molecules using multiple singly labeled probes. Nat Methods 2008; 5: 877-879.

8 Strunz M, Simon LM, Ansari M, et al. Longitudinal single cell transcriptomics reveals Krt8+ alveolar epithelial progenitors in lung regeneration. BioRxiv 2019; preprint [https://doi.org/10.1101/705244].

9 La Manno G, Soldatov R, Zeisel A, et al. RNA velocity of single cells. Nature 2018; 560: 494-498.

10 Bonnelykke K, Sleiman P, Nielsen K, et al. A genome-wide association study identifies CDHR3 as a susceptibility locus for early childhood asthma with severe exacerbations. Nat Genet 2014; 46: 51-55.

11 Bonnelykke K, Coleman AT, Evans MD, et al. Cadherin-related family member 3 genetics and rhinovirus C respiratory illnesses. Am J Respir Crit Care Med 2018; 197: 589-594.

12 Bogaert D, De Groot R, Hermans PW. Streptococcus pneumoniae colonisation: the key to pneumococcal disease. Lancet Infect Dis 2004; 4: 144-154.

13 Jochems SP, Marcon F, Carniel BF, et al. Inflammation induced by influenza virus impairs human innate immune control of pneumococcus. Nat Immunol 2018; 19: 1299-1308.

14 Jochems SP, de Ruiter K, Solorzano C, et al. Innate and adaptive nasal mucosal immune responses following experimental human pneumococcal colonization. J Clin Invest 2019; 130: 4523-4538.

15 Kaufmann E, Sanz J, Dunn JL, et al. BCG educates hematopoietic stem cells to generate protective innate immunity against tuberculosis. Cell 2018; 172: 176-190.

16 Netea MG, Joosten LA, Latz E, et al. Trained immunity: a program of innate immune memory in health and disease. Science 2016; 352: aaf1098.

17 Netea MG, Quintin J, van der Meer JW. Trained immunity: a memory for innate host defense. Cell Host Microbe 2011; 9: 355-361.

18 Nemes E, Geldenhuys H, Rozot V, et al. Prevention of M. tuberculosis infection with H4:IC31 vaccine or BCG revaccination. N Engl J Med 2018; 379: 138-149.

19 Butkeviciute E, Jones CE, Smith SG. Heterologous effects of infant BCG vaccination: potential mechanisms of immunity. Future Microbiol 2018; 13: 1193-1208. 
20 Tanner R, Villarreal-Ramos B, Vordermeier HM, et al. The humoral immune response to BCG vaccination. Front Immunol 2019; 10: 1317.

21 Quintin J, Cheng SC, van der Meer JW, et al. Innate immune memory: towards a better understanding of host defense mechanisms. Curr Opin Immunol 2014; 29: 1-7.

22 Sher NA, Chaparas SD, Greenberg LE, et al. Effects of BCG, Corynebacterium parvum, and methanol-extration residue in the reduction of mortality from Staphylococcus aureus and Candida albicans infections in immunosuppressed mice. Infect Immun 1975; 12: 1325-1330.

23 van 't Wout JW, Poell R, van Furth R. The role of BCG/PPD-activated macrophages in resistance against systemic candidiasis in mice. Scand J Immunol 1992; 36: 713-719.

24 Benn CS, Netea MG, Selin LK, et al. A small jab - a big effect: nonspecific immunomodulation by vaccines. Trends Immunol 2013; 34: 431-439.

25 Garly ML, Martins CL, Bale C, et al. BCG scar and positive tuberculin reaction associated with reduced child mortality in West Africa. A non-specific beneficial effect of BCG? Vaccine 2003; 21: 2782-2790.

26 Yao Y, Jeyanathan M, Haddadi S, et al. Induction of autonomous memory alveolar macrophages requires $\mathrm{T}$ cell help and is critical to trained immunity. Cell 2018; 175: 1634-1650.

27 Bauer M, Weis S, Netea MG, et al. Remembering pathogen dose: long-term adaptation in innate immunity. Trends Immunol 2018; 39: 438-445.

28 Bekkering S, Arts RJW, Novakovic B, et al. Metabolic induction of trained immunity through the mevalonate pathway. Cell 2018; 172: 135-146.

29 Mulder WJM, Ochando J, Joosten LAB, et al. Therapeutic targeting of trained immunity. Nat Rev Drug Discov 2019; 18: 553-566.

30 Viniol C, Vogelmeier CF. Exacerbations of COPD. Eur Respir Rev 2018; 27: 170103.

31 Papi A, Bellettato CM, Braccioni F, et al. Infections and airway inflammation in chronic obstructive pulmonary disease severe exacerbations. Am J Respir Crit Care Med 2006; 173: 1114-1121.

32 Calderazzo MA, Trujillo-Torralbo MB, Finney LJ, et al. Inflammation and infections in unreported chronic obstructive pulmonary disease exacerbations. Int J Chron Obstruct Pulmon Dis 2019; 14: 823-832.

33 Mallia P, Webber J, Gill SK, et al. Role of airway glucose in bacterial infections in patients with chronic obstructive pulmonary disease. J Allergy Clin Immunol 2018; 142: 815-823.

34 Molyneaux PL, Mallia P, Cox MJ, et al. Outgrowth of the bacterial airway microbiome after rhinovirus exacerbation of chronic obstructive pulmonary disease. Am J Respir Crit Care Med 2013; 188: 1224-1231.

35 Singanayagam A, Glanville N, Girkin JL, et al. Corticosteroid suppression of antiviral immunity increases bacterial loads and mucus production in COPD exacerbations. Nat Commun 2018; 9: 2229.

36 Singanayagam A, Glanville N, Cuthbertson L, et al. Inhaled corticosteroid suppression of cathelicidin drives dysbiosis and bacterial infection in chronic obstructive pulmonary disease. Sci Transl Med 2019; 11: eaav3879.

37 Manuck TA, Levy PT, Gyamfi-Bannerman C, et al. Prenatal and perinatal determinants of lung health and disease in early life: a National Heart, Lung, and Blood Institute Workshop Report. JAMA Pediatr 2016; 170 : e154577.

38 Kotecha SJ, Watkins WJ, Heron J, et al. Spirometric lung function in school-age children: effect of intrauterine growth retardation and catch-up growth. Am J Respir Crit Care Med 2010; 181: 969-974.

39 Zeitlin J, El Ayoubi M, Jarreau PH, et al. Impact of fetal growth restriction on mortality and morbidity in a very preterm birth cohort. J Pediatr 2010; 157: 733-739.

40 Boghossian NS, Geraci M, Edwards EM, et al. In-hospital outcomes in large for gestational age infants at 22-29 weeks of gestation. J Pediatr 2018; 198: 174-180.

41 Metsala J, Kilkkinen A, Kaila M, et al. Perinatal factors and the risk of asthma in childhood - a population-based register study in Finland. Am J Epidemiol 2008; 168: 170-178.

42 Rautava L, Hakkinen U, Korvenranta E, et al. Health and the use of health care services in 5-year-old very-low-birth-weight infants. Acta Paediatr 2010; 99: 1073-1079.

43 Twilhaar ES, de Kieviet JF, Aarnoudse-Moens CS, et al. Academic performance of children born preterm: a meta-analysis and meta-regression. Arch Dis Child Fetal Neonatal Ed 2018; 103: F322-F330.

44 Kallapur SG, Kramer BW, Knox CL, et al. Chronic fetal exposure to Ureaplasma parvum suppresses innate immune responses in sheep. J Immunol 2011; 187: 2688-2695.

45 Kemp MW, Saito M, Schmidt AF, et al. The duration of fetal antenatal steroid exposure determines the durability of preterm ovine lung maturation. Am J Obstet Gynecol 2020; 222: 183.

46 Sharma G, Goodwin J. Effect of aging on respiratory system physiology and immunology. Clin Interv Aging 2006 1: 253-260.

47 Forster K, Ertl-Wagner B, Ehrhardt H, et al. Altered relaxation times in MRI indicate bronchopulmonary dysplasia. Thorax 2020; 75: 184-187.

48 Barbera JA, Peinado VI, Santos S. Pulmonary hypertension in chronic obstructive pulmonary disease. Eur Respir J 2003; 21: 892-905.

49 Carrero D, Soria-Valles C, Lopez-Otin C. Hallmarks of progeroid syndromes: lessons from mice and reprogrammed cells. Dis Model Mech 2016; 9: 719-735.

50 Meiners S, Hilgendorff A. Early injury of the neonatal lung contributes to premature lung aging: a hypothesis. Mol Cell Pediatr 2016; 3: 24.

51 Oak P, Pritzke T, Thiel I, et al. Attenuated PDGF signaling drives alveolar and microvascular defects in neonatal chronic lung disease. EMBO Mol Med 2017; 9: 1504-1520.

52 Angata T, Ishii T, Motegi T, et al. Loss of Siglec-14 reduces the risk of chronic obstructive pulmonary disease exacerbation. Cell Mol Life Sci 2013; 70: 3199-3210.

53 Mobius MA, Rudiger M. Mesenchymal stromal cells in the development and therapy of bronchopulmonary dysplasia. Mol Cell Pediatr 2016; 3: 18.

54 Ghosh A, Coakley RC, Mascenik T, et al. Chronic e-cigarette exposure alters the human bronchial epithelial proteome. Am J Respir Crit Care Med 2018; 198: 67-76.

55 Smeets CC, Codd V, Samani NJ, et al. Leukocyte telomere length in young adults born preterm: support for accelerated biological ageing. PLoS One 2015; 10: e0143951.

56 Saglani S, Lui S, Ullmann N, et al. IL-33 promotes airway remodeling in pediatric patients with severe steroid-resistant asthma. J Allergy Clin Immunol 2013; 132: 676-685. 
57 Nagakumar P, Puttur F, Gregory LG, et al. Pulmonary type-2 innate lymphoid cells in paediatric severe asthma: phenotype and response to steroids. Eur Respir J 2019; 54: 1801809.

58 Puttur F, Denney L, Gregory LG, et al. Pulmonary environmental cues drive group 2 innate lymphoid cell dynamics in mice and humans. Sci Immunol 2019; 4: eaav7638.

59 Pattaroni C, Watzenboeck ML, Schneidegger S, et al. Early-life formation of the microbial and immunological environment of the human airways. Cell Host Microbe 2018; 24: 857-865.

60 de Koff EM, Pattaroni C, Marsland BJ, et al. The early-life microbiome: the key to respiratory health? In: Cox MJ, Ege MJ, von Mutius E, eds. The Lung Microbiome (ERS Monograph). Sheffield, European Respiratory Society, 2019; pp. 67-87.

61 Bosch A, de Steenhuijsen Piters WAA, van Houten MA, et al. Maturation of the infant respiratory microbiota. environmental drivers, and health consequences. A prospective cohort study. Am J Respir Crit Care Med 2017; 196: $1582-1590$.

62 Man WH, Clerc M, de Steenhuijsen Piters WAA, et al. Loss of microbial topography between oral and nasopharyngeal microbiota and development of respiratory infections early in life. Am J Respir Crit Care Med 2019; 200: 760-770.

63 Man WH, van Houten MA, Merelle ME, et al. Bacterial and viral respiratory tract microbiota and host characteristics in children with lower respiratory tract infections: a matched case-control study. Lancet Respir Med 2019; 7: 417-426.

64 Reinke SN, Gallart-Ayala H, Gomez C, et al. Metabolomics analysis identifies different metabotypes of asthma severity. Eur Respir J 2017; 49: 1601740.

65 Naz S, Kolmert J, Yang M, et al. Metabolomics analysis identifies sex-associated metabotypes of oxidative stress and the autotaxin-lysoPA axis in COPD. Eur Respir J 2017; 49: 1602322.

66 Ito K, Barnes PJ. COPD as a disease of accelerated lung aging. Chest 2009; 135: 173-180.

67 Kumar M, Seeger W, Voswinckel R. Senescence-associated secretory phenotype and its possible role in chronic obstructive pulmonary disease. Am J Respir Cell Mol Biol 2014; 51: 323-333.

68 Justice JN, Nambiar AM, Tchkonia T, et al. Senolytics in idiopathic pulmonary fibrosis: results from a first-in-human, open-label, pilot study. EBioMedicine 2019; 40: 554-563.

69 Cloonan SM, Choi AM. Mitochondria in lung disease. J Clin Invest 2016; 126: 809-820.

70 Wiegman $\mathrm{CH}$, Michaeloudes $\mathrm{C}$, Haji G, et al. Oxidative stress-induced mitochondrial dysfunction drives inflammation and airway smooth muscle remodeling in patients with chronic obstructive pulmonary disease. J Allergy Clin Immunol 2015; 136: 769-780.

71 Ryter SW, Rosas IO, Owen CA, et al. Mitochondrial dysfunction as a pathogenic mediator of chronic obstructive pulmonary disease and idiopathic pulmonary fibrosis. Ann Am Thorac Soc 2018; 15: Suppl. 4, S266-S272.

72 Beckett EL, Stevens RL, Jarnicki AG, et al. A new short-term mouse model of chronic obstructive pulmonary disease identifies a role for mast cell tryptase in pathogenesis. J Allergy Clin Immunol 2013; 131: 752-762.

73 Murphy MP. How mitochondria produce reactive oxygen species. Biochem J 2009; 417: 1-13.

74 Xie ZZ, Liu Y, Bian JS. Hydrogen sulfide and cellular redox homeostasis. Oxid Med Cell Longev 2016; 2016: 6043038.

75 Sun Y, Wang K, Li MX, et al. Metabolic changes of $\mathrm{H}_{2} \mathrm{~S}$ in smokers and patients of COPD which might involve in inflammation, oxidative stress and steroid sensitivity. Sci Rep 2015; 5: 14971.

76 Chen YH, Yao WZ, Geng B, et al. Endogenous hydrogen sulfide in patients with COPD. Chest 2005; 128 : 3205-3211.

77 Mills EL, Ryan DG, Prag HA, et al. Itaconate is an anti-inflammatory metabolite that activates Nrf2 via alkylation of KEAP1. Nature 2018: 556: 113-117.

78 Hara $\mathrm{H}$, Araya J, Ito S, et al. Mitochondrial fragmentation in cigarette smoke-induced bronchial epithelial cell senescence. Am J Physiol Lung Cell Mol Physiol 2013; 305: L737-L746.

79 Belchamber KBR, Singh R, Batista CM, et al. Defective bacterial phagocytosis is associated with dysfunctional mitochondria in COPD macrophages. Eur Respir J 2019; 54: 1802244.

80 Spees JL, Olson SD, Whitney MJ, et al. Mitochondrial transfer between cells can rescue aerobic respiration. Proc Natl Acad Sci USA 2006; 103: 1283-1288.

81 Jackson MV, Morrison TJ, Doherty DF, et al. Mitochondrial transfer via tunneling nanotubes is an important mechanism by which mesenchymal stem cells enhance macrophage phagocytosis in the in vitro and in vivo models of ARDS. Stem Cells 2016; 34: 2210-2223.

82 Morrison TJ, Jackson MV, Cunningham EK, et al. Mesenchymal stromal cells modulate macrophages in clinically relevant lung injury models by extracellular vesicle mitochondrial transfer. Am J Respir Crit Care Med 2017; 196 $1275-1286$. 